\title{
Coexistent molar pregnancy with a normal fetus-a rare presentation of gestational trophoblastic disease
}

\section{Catarina J. Nascimento*, Mariana Veiga, Ana Rita Silva, Joana Cominho}

Department of Gynecology and Obstetrics, Hospital de Cascais Dr. José de Almeida, Cascais, Portugal

Received: 14 August 2021

Accepted: 13 September 2021

\author{
*Correspondence: \\ Dr. Catarina J. Nascimento, \\ E-mail: Catarina.nascimento@gmail.com
}

Copyright: (C) the author(s), publisher and licensee Medip Academy. This is an open-access article distributed under the terms of the Creative Commons Attribution Non-Commercial License, which permits unrestricted non-commercial use, distribution, and reproduction in any medium, provided the original work is properly cited.

\begin{abstract}
A coexistent molar pregnancy with a normal fetus is rare, with an incidence of 1 in 22.000 to 100.000 pregnancies-only 200 cases reported in the last two decades. The ultrasound is essential for an earlier diagnosis, and the management of these cases is challenging due to the increased risk of obstetrics complications and the possibility of posterior gestational trophoblastic neoplasia. Here we describe a 33-year-old healthy woman with a first-trimester twin pregnancy, presented with a normal fetus and a heterogeneous and vacuolar structure suggestive of complete hydatidiform mole. The pregnancy was interrupted, and a histological diagnosis confirmed complete hydatidiform mole in dichorionic/diamniotic twin pregnancy at 14 weeks. Molar twin pregnancy is a rare condition, and do not exist any consensus protocol to guide the clinical approach, so the decision to continue the pregnancy depends on the couple's desire and maternal and fetal complications.
\end{abstract}

Keywords: Molar pregnancy, Trophoblastic disease, Hydatidiform mole, Twin pregnancy, Ultrasound, Case report

\section{INTRODUCTION}

Molar pregnancy belongs to a group of gestational trophoblastic diseases derived from the placenta.

Although largely unclear, the aetiology of gestational trophoblastic disease likely involves genetic abnormalities involved in fertilization. ${ }^{1,2}$

Coexistent molar pregnancy with a normal fetus is a scarce situation, with most of the cases diagnosed antepartum by ultrasound findings of a complex, cystic placental component distinct from the fetoplacental unit. ${ }^{3}$

It has an incidence of 1 in 22.000 to 100.000 pregnancieswith only about 200 cases reported in past two decades. ${ }^{4}$

Management of these cases is challenging due to the increased risk of antenatal and perinatal complications and the risk of developing gestational trophoblastic neoplasia after delivery. ${ }^{2}$
It is essential to provide proper patient counselling regarding pregnancy continuation and alert for maternal and fetal risks.

\section{CASE REPORT}

A 33-year-old healthy, ARh+, leucodermic woman with a previous normal pregnancy and a healthy child was sent from a primary health care unit for a prenatal diagnosis hospital appointment with a seven-week twin pregnancy.

At 13 weeks and 3 days, the first-trimester ultrasound identified a normal live fetus (Figure 1 A) with negative ultrasound aneuploidies markers (Figure 1 B) and an anterior placenta.

It was noted that a heterogeneous structure, with multiple vacuolar image, without the presence of fetal parts, was located on the supracervical left lateral side with $40 \times 27$ $\mathrm{mm}$ suggestive of hydatidiform mole (Figure $2 \mathrm{~A}$ and $\mathrm{B}$ ). 


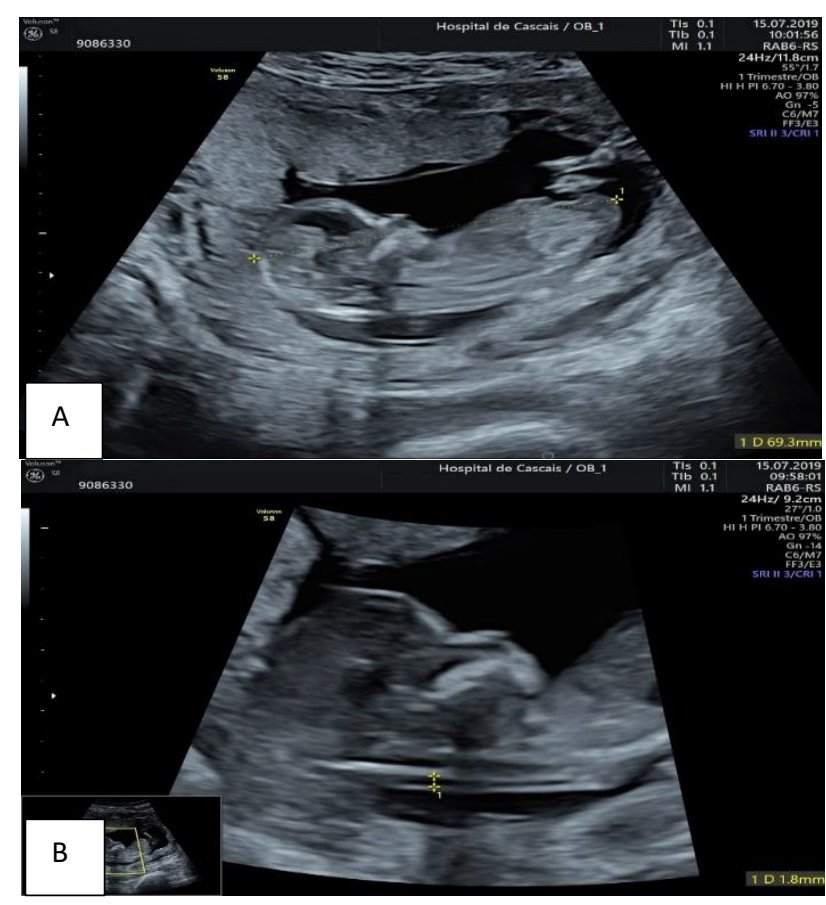

Figure 1 (A and B): First-trimester ultrasound; 13 weeks and 3 days normal fetus and normal placenta and nuchal translucency of $1.8 \mathrm{~mm}$.

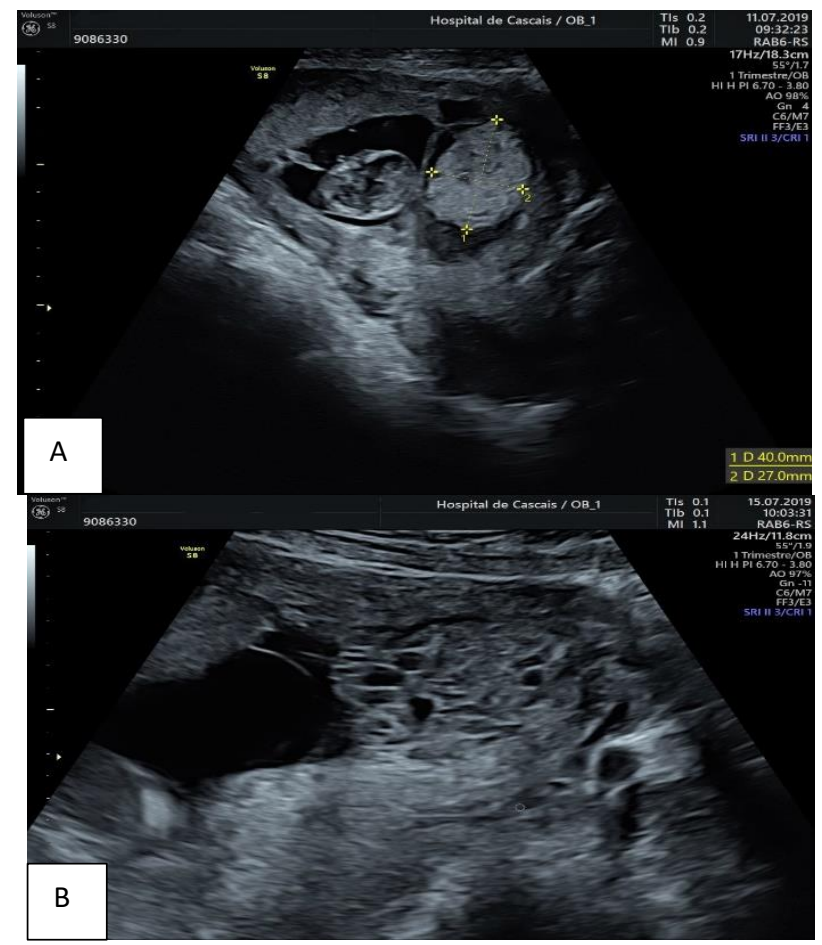

Figure 2 (A and B): Heterogeneous structure with multiple vacuolar image, $40 \times 27 \mathrm{~mm}$, on the right, and fetus on the left and multiple vacuolar image, without presence of fetal parts-hydatidiform mole.

Analytically, the highlight 551592 IU/L of beta-hCG, with a normal thyroid, hepatic, renal function and chest Xray without changes. The couple was duly informed about the complications associated with the suspected pathology and was given the possibility to interrupt. The couple thought and asked to interrupt the pregnancy. The interruption took place one week after the decision. The $200 \mathrm{mg}$ of mifepristone were given 48 hours before, followed by the administration of misoprostol protocol. The expulsion of the fetus and vesicular placenta fragments (Figure 3) took place in less than 24 hours. Subsequently, uterine cavity evacuation was performed with oxytocin perfusion.

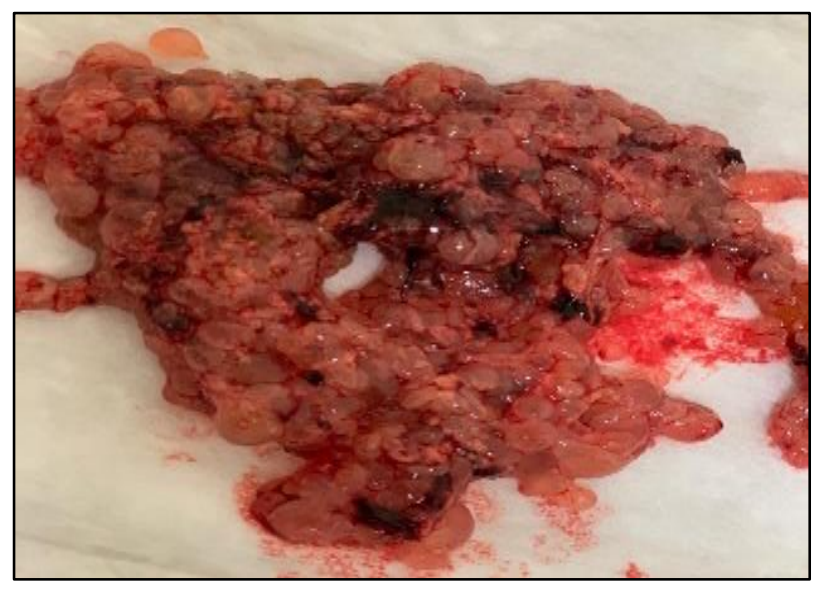

Figure 3: Macroscopic vesicular placenta fragments after evacuation.

Histological diagnosis and autopsy confirmed morphological changes consistent with complete hydatidiform mole in dichorionic/diamniotic twin pregnancy compatible with 14 weeks. Autopsy describe a dichorionic/diamniotic twin pregnancy with first yolk sac with fetus and placenta and second with molar product. Signs of anoxia are documented, probably secondary to the medical interruption of pregnancy. Malformations and developmental disturbances are not documented. The placenta histology describes a dichorionic/diamniotic twin pregnancy placenta, one of which corresponds to a complete hydatidiform mole. The non-molar placenta, consistent with the second-trimester pregnancy, with vascular congestion of the three cord vessels and villous chorion with deposits of perivilositary fibrinoid substance and haemorrhage areas.

The patient was kept under surveillance in the firsttrimester pathology appointment, always asymptomatic with weekly beta-hCG measurement, which was negative after 8 weeks of evacuation. She opted for hormonal contraception with desogestrel and maintained follow-up with monthly measurements until 6 months after the first negative value.

\section{DISCUSSION}

Gestational trophoblastic disease describes a spectrum of neoplastic conditions derived from the placenta, including hydatidiform moles, post-molar gestational trophoblastic neoplasia, gestational choriocarcinoma, placental site 
trophoblastic tumour, and epithelioid trophoblastic tumour. ${ }^{2,3}$

A combined algorithm using beta-hCG, clinical history, examination and imaging is required to diagnose hydatidiform mole. At the ultrasound, molar tissue typically is identified as a diffuse mixed echogenic and vesicular pattern replacing the placenta, traditionally labelled a "snowstorm appearance". 3

Complete hydatidiform moles do not have fetal tissues, and the chorionic villi exhibit generalized hydatidiform swelling and diffuse trophoblastic hyperplasia. In this condition, cytogenetic studies show a 46XX karyotype and molar chromosomes are entire of paternal origin. Recent evidence suggests that complete moles may develop from the post-zygotic diploidization of a triploid conception instead of an anucleate ovum, which has been fertilized by a haploid sperm that duplicates its own chromosomes. ${ }^{2}$

Approximately 5\% of complete hydatidiform moles have a 46, XY androgenetic genome, indicating that dispermic fertilization occurs in some. ${ }^{3}$

Twin pregnancy with a fetus and hydatidiform mole is rare, and ultrasound is the first exam to perform that has more accuracy at the end of the first trimester. ${ }^{6-8}$ The current ultrasound improvements have allowed an earlier diagnosis with findings of a complex, cystic placental component distinct from the fetoplacental unit. ${ }^{9}$ Sometimes is a challenge to differentiate a twin pregnancy with a complete mole from placentomegaly, and magnetic resonance imaging can be an essential diagnostic tool. ${ }^{8}$

There are no clear guidelines for managing patients with coexistent hydatidiform mole and fetus suspected by ultrasonography. It is crucial to exclude retroplacental hematoma, other placental abnormalities, degenerating myoma and thoroughly evaluate the fetoplacental unit for evidence of a partial mole or gross fetal malformations. ${ }^{3}$

Three disease entities must be considered in the differential diagnosis: a singleton pregnancy containing a partial mole and one viable fetus; a twin pregnancy with a complete mole and one viable twin with a separate placenta; and a combination of a partial mole with a twin in one amniotic sac, and one "normal" twin in the other. 9 Another identity that needs attention is placental mesenchymal dysplasia, characterized by the presence of placentomegaly with multiple prominent cysts with hypoechoic areas, dilated chorionic vessels and without atypical trophoblastic proliferation. This condition can exist with a viable fetus, and pregnancy may be allowed to progress. ${ }^{2}$ However, spontaneous preterm delivery (33\%), intrauterine growth restriction $(33 \%)$ and intrauterine fetal death $(13 \%)$ were reported. ${ }^{8}$

The karyotype analysis of the suspected placenta and the fetus and the immuno-cytochemical analysis of the p57kip2 gene are complementary exams for differential diagnosis of these pathologies. The complete hydatidiform mole has an androgenetic origin and does not express p57kip2. ${ }^{6,7}$

Twin pregnancy with a complete hydatidiform mole has a higher risk of maternal and fetal complications as compared to a singleton pregnancy or a non-molar twin pregnancy. ${ }^{10,11}$ These include preeclampsia, eclampsia, antepartum bleeding, placenta previa, preterm premature rupture of the membranes and preterm labour. The fetal complications comprise spontaneous abortion, intrauterine growth restriction and fetal death. ${ }^{9}$

A recent review article, with 72 cases of twin pregnancy with complete hydatidiform, demonstrated that $67 \%$ of the women had an antepartum haemorrhage, 30\% developed early preeclampsia and $1.4 \%$ maternal death. ${ }^{2,12}$ In another review article with 206 cases, Suksai et al reported a pregnancy termination rate of $32 \%$ due to maternal complications and $18 \%$ of fetal loss in women's that want to continue their pregnancies. ${ }^{2,10}$

Another complication associated with this pathology is an increased risk of developing persistent gestational trophoblastic disease. Compared with singleton hydatidiform moles, twin pregnancy with a fetus and mole has an increased risk for post-molar GTN, and a higher proportion of patients develop high-risk GTN requiring multi-agent chemotherapy. ${ }^{3}$

The absolute risk of gestational neoplasm of the trophoblast is similar in women who choose early termination of pregnancy than those who prefer to maintain the pregnancy, thus showing that the risk of persistent trophoblastic disease does not increase with advancing gestational age. ${ }^{11,13,9}$

The most common metastatic site of gestational trophoblastic disease is the lungs, which are affected in over $80 \%$ of patients. The second most common site of metastasis is the vagina, around $30 \%$ of cases. ${ }^{14}$ Earlystage gestational trophoblastic neoplasia is often cured with single-agent chemotherapy, but the advanced stage needs multi-agent combination chemotherapeutic regimens. ${ }^{14}$ In most cases, gestational trophoblastic neoplasia following twin pregnancy with a complete mole can be adequately treated with single-agent methotrexate. ${ }^{2,10}$

If the diagnosis is still suspected and continuation of pregnancy is desired, fetal karyotype should be obtained, chest radiography should be performed to screen for metastases, and serial serum beta-hCG values should be followed. ${ }^{3}$

If the fetal karyotype is normal, major fetal malformations are excluded by ultrasonography, and if there is no evidence of metastatic disease, it is reasonable to allow the pregnancy to continue unless pregnancy-related complications force delivery. After delivery, the placenta 
should be histologically evaluated, and the patient should be followed closely with serial beta-hCG values, as one would with a singleton hydatidiform mole. ${ }^{3}$

Predictors of good pregnancy outcomes include lower beta-hCG levels at diagnosis $(<400000 \mathrm{mIU} / \mathrm{mL})$, later gestational age at diagnosis, and a lack of antenatal maternal medical complications that may achieve a favourable obstetric outcome. ${ }^{2,6}$ It is crucial to have close surveillance of maternal and fetal status. Furthermore, the postpartum follow-up is critical to detect any signs of postmolar persistent trophoblastic disease. ${ }^{2,3}$

There are no specific guidelines for monitoring twin pregnancy with complete hydatidiform. However, recommendations for general molar pregnancy should be followed:

After evacuation, the value of serum beta-hCG levels is obtained weekly in all patients until the levels remain undetectable. The international federation of gynecology and obstetrics (FIGO) recommends following patients with molar pregnancies with beta-hCG levels every one to two weeks until beta-hCG normalization and then monthly. In patients with a complete mole, confirmatory normal beta-hCG levels are needed for six months before discontinuing beta-hCG monitoring. ${ }^{15}$

Patients with hydatidiform mole must be advised to use reliable contraception during the entire period of postoperative beta-hCG monitoring.

\section{CONCLUSION}

In summary, given the complications presented, many couples choose to interrupt the pregnancy, as happened in the case presented. The decision to continue the pregnancy always depends on maternal and fetal complications, obstetric past and above all, the couple's desire. The postinterruption period must be closely monitored, with adequate follow-up to detect early persistent trophoblast disease. Women with a history of gestational trophoblastic disease are at increased risk of developing a subsequent gestational trophoblastic disease, and therefore their future pregnancies require careful monitoring.

Funding: No funding sources Conflict of interest: None declared

Ethical approval: Not required

\section{REFERENCES}

1. Melamed A, Gockley AA, Joseph NT, Sun SY, Clapp MA, Goldstein DP et al. Effect of race/ethnicity on risk of complete and partial molar pregnancy after adjustment for age. Gynecol Oncol. 2016;143(1):73-6.

2. Lipi LB, Philp L, Goodman AK. A challenging case of twin pregnancy with complete hydatidiform mole and co-existing normal live fetus-A case report and review of the literature. Gynecologic Oncol Rep. 2020;31:100519.

3. DiSaia P. Creasman W, Mannell R, McMeekin S, Mutch D. In Clinical Gynecologic Oncology, $9^{\text {th }}$ edition, Elsevier. 2017.

4. Vimercati A, Gennaro AC, De Cobuzzi I, Grasso S, Abruzzese M, Fascilla F et al. Two cases of complete hydatidiform mole and coexistent live fetus. J Prenatal Med. 2013;7(1):1-4.

5. Altieri A, Franceschi S, Ferlay J, Smith J, La Vecchia C. Epidemiology and etiology of gestational trophoblastic diseases. Lancet Oncol. 2003;4(11):670.

6. Braga A, Obeica B, Werner H, Sun SY, Amim Junior J, Filho JR et al. A twin pregnancy with a hydatidiform mole and a coexisting live fetus: prenatal diagnosis, treatment, and follow-up. J Ultrason 2017;17:299-305.

7. Madi JM, Braga A, Paganella MP, Litvin IE, Da Ros Wendland EM. Accuracy of p57KIP2 compared with genotyping for the diagnosis of complete hydatidiform mole: Protocol for a systematic review and metaanalysis. Syst Rev. 2016;5:169.

8. Marusik C, Frykholm C, Ericson K, Wikström J, Axelsson O. Diagnosis of placental mesenchymal dysplasia with magnetic resonance imaging. Ultrasound Obstet Gynecol. 2017;49:410-12.

9. Freis A, Elsasser M, Sohn C, Fluhr H. Twin Pregnancy with One Fetus and One Complete Mole-A Case Report. Geburtsh Frauenheilk. 2016;76:819-22.

10. Suksai M, Suwanrath C, Kor-anantakul O, Geater A, Hanprasertpong $\mathrm{T}$ et al. Complete hydatidiform mole with co-existing fetus: predictors of live birth. Eur J Obstet Gynecol. 2017;212:1-8.

11. Sebire NJ, Foskett M, Paradinas FJ, Fisher RA, Francis RJ, Short D et al. Outcome of twin pregnancies with complete hydatidiform mole and healthy cotwin. Lancet. 2002;359:2165-6.

12. Lin LH, Maestá I, Braga A, Sun SY, Fushida K, Francisco RPV et al. Multiple pregnancies with complete mole and coexisting normal fetus in North and South America: a retrospective multicenter cohort and literature review. Gynecol Oncol. 2017;145:88-95.

13. Giorgione V, Cavoretto P, Cormio G, Valsecchi L, Vimercati A, De Gennaro A et al. Prenatal diagnosis of twin pregnancies with complete hydatidiform mole and coexistent normal fetus: a series of 13 cases. Gynecol Obstet Invest. 2017;82:404-40.

14. Deng L, Yan $\mathrm{X}$, Zhang J, Wu T. Combination chemotherapy for high-risk gestational trophoblastic tumour. Cochrane Database Syst Rev. 2009;15:CD005196.

15. Ngan HYS, Seckl MJ, Berkowitz RS, Xiang Y, Golfier F, Sekharan PK et al. Update on the diagnosis and management of gestational trophoblastic disease. Int J Gynaecol Obstet. 2018;143(2):79.

Cite this article as: Nascimento CJ, Veiga M, Silva AR, Cominho J. Coexistent molar pregnancy with a normal fetus-a rare presentation of gestational trophoblastic disease. Int J Reprod Contracept Obstet Gynecol 2021;10:3962-5. 